\title{
ITERATIVE QR DECOMPOSTION CHANNEL ESTIMATION FOR MIMO-OFDM SYSTEMS
}

\author{
R. Prakash Kumar', I Raghu' ${ }^{2}$, M. Vinod Kumar Reddy ${ }^{3}$ \\ 1, 2, ${ }^{3}$ Assistant Professor, ECE Department, CVR College of Engineering, AP, India, \\ prakash.rachmagdu@gmail.com,raghu.indraganti303@gmail.com,vinodkumarreddy488@gmail.com
}

\begin{abstract}
Channel estimation algorithms have a key role in signal detection in MIMO-OFDM systems. In this system, the number of channel components which need to be estimated is much more than conventional SISO wireless systems. Consequently, the computational process of channel estimation is highly intensive. In addition, the high performance channel estimation algorithms mostly suffer from high computational complexity. In the other words, the system undergoes intensive computations if high performance efficiency is desired. However, there is an alternative solution to achieve both high performance efficiency and relatively low level of computational complexity. In this solution, high efficient channel estimation is firstly designed, and then it is simplified using alternative mathematical expressions. In this paper, Iterative channel estimation based on QR decomposition for MIMO-OFDM systems is proposed. From simulation results, the iterative QRD channel estimation algorithm can provide better mean-square-error and bit error rate performance than conventional methods.
\end{abstract}

Index Terms: MIMO, OFDM, QRD, Least squre Channel estimation

\section{INTRODUCTION}

Orthogonal frequency division multiplexing (OFDM) technology has been used widely in many wireless communication systems, such as digital audio broadcasting (DAB), digital video broadcasting (DVB), wireless local area network (WLAN), asymmetric digital subscriber loop (ADSL), and future 4G systems. OFDM systems can provide higher bandwidth efficiency and achieve higher data throughput. In order to enhance the data rate, multi-antenna technique is applied to existing systems. Multiple-input multiple-output (MIMO) communication refers to wireless communication systems using an array of antennas (i.e. multiple antennas) at either the transmitter or the receiver. Multiplexing would cause interference, but MIMO systems use smart selection and/or combining techniques at the receiving end to transmit more information and to improve signal quality.

Therefore, MIMO OFDM systems are regarded as attractive systems for high speed transmission. Hence, the integration of these two technologies has the potential to meet the ever growing demands of future communication systems [1]. If space-time coding is used at the transmitter, the channel knowledge is required at the receiver to decode the transmitted symbols. Therefore, accurate channel estimation plays a key role in data detection especially in MIMO-OFDM system where the number of channel coefficients is $\mathrm{M} \times \mathrm{N}$ time more than SISO system. ( $\mathrm{M}$ and $\mathrm{N}$ are the number of transmitted and received antenna respectively). Technically, there are four types of channel estimation [2]; training-based, blind, semiblind and data-aided channel estimation. In wireless communications, signals are always distorted by channel. The wireless channel is time or location variant the channel state information to compensate the channel distortion. Pilot signals can be spaced separated in the transmitted symbols. In the receiver, the channel impulse response can be estimated at the positions of pilot signals. The other channel information at the data signals can be obtained by interpolating the estimated channel impulse response. However, error caused by channel interpolation cannot be avoided. A good channel estimation method can provide higher reliable data detection.

In this paper, we proposed iterative channel estimation based on QR Decomposition for MIMO OFDM systems. The aim of this paper is to investigate the effectiveness of $\mathrm{QRD}(\mathrm{QR}$ decomposition) to reduce the computational complexity of channel estimation algorithms in MIMO-OFDM system, and design high performance channel estimation for this system by using iterative technique.

\section{SYSTEM MODEL}

Figure.1 shows the basic model of MIMO-OFDM system with $\mathrm{M}$ and $\mathrm{Nr}$ number of antenna at the transmitter and receiver respectively. In this model, MIMO transmission is assumed to be OSTBC (Orthogonal Space-Time Block Coded). Therefore the block of user information after mapping in MPSK modulator is coded by the MIMO-STBC encoder with the matrix dimension of $\mathrm{P} \times \mathrm{M}$. Where $\mathrm{P}$ is the number of time 
interval needed to transmit this matrix by $\mathrm{M}$ number of transmit antenna.

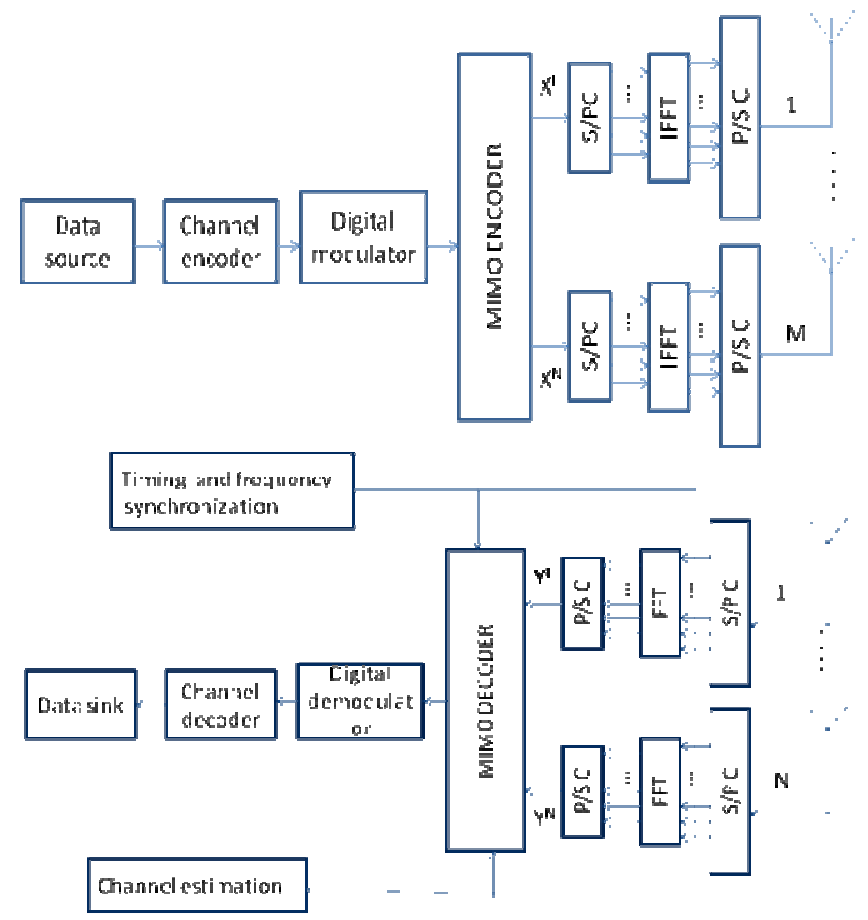

$\mathrm{S} / \mathrm{P} \mathrm{C}=$ Serial to Parallel Converter

$\mathrm{P} / \mathrm{S} \mathrm{C}=$ Parallel to Serial Converter

$\mathrm{FFT}=$ Fast Fourier Transform

IFFT $=$ Inverse Fast Fourier Transform

Figure 1.Block diagram of an MIMO-OFDM transceiver

If a column of encoded matrix which enter to the OFDM block is $(\mathrm{X} 1, \mathrm{X} 2, \ldots, \mathrm{Xm}) \mathrm{T}$ in frequency domain then the output of OFDM module will be $(\mathrm{x} 1, \mathrm{x} 2, \ldots, \mathrm{xm}) \mathrm{T}$ in time domain. Each element of encoded matrix Xk before OFDM module has a length of $\mathrm{N}=64$ symbols while after OFDM module change to $\mathrm{xk}$ in time domain with the length of 80 symbols. i.e received signal after distortion by frequency selective channel and AWG noise at antenna $\mathrm{j}$ from antenna $\mathrm{i}$ can be represented by "Equation (1)".

$$
y^{j i}(n)=\sum_{l=0}^{L-1} h_{l}^{j i}(n) \cdot x^{i}(n-l)+w^{j}(n), i=1,2, \ldots . M
$$

Where is $h_{l}^{j i}(n)$ is lth channel coefficient between received antenna $j$ and transmitted antenna $i$ at time $n$. WJ(n) is AWGN with zero mean and variance one. The "Equation (1)" in vector form can be rewritten by "Equation (2), (3), (4), (5)".

Received signal vector at time interval $t$ by antenna $j=1$, $2, \ldots, \mathrm{Nr}$ can be represented as

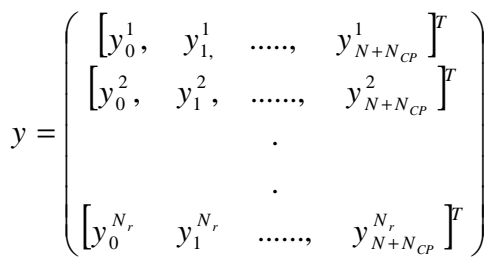

$$
\begin{aligned}
& y=\left(\begin{array}{ccccc}
\sum_{i=1}^{M} \text { toeplitz } & \left(h^{i 1}\right) \cdot\left[\begin{array}{llll}
x_{0}^{i}, & x_{1}^{i}, & \ldots . ., & x_{N+N_{C P}}^{i}
\end{array}\right] \\
\sum_{i=1}^{M} \text { toeplitz } & \left(h^{i 2}\right)\left[\begin{array}{llll}
x_{0}^{i}, & x_{1}^{i}, & \ldots \ldots . ., & x_{N+N_{C P}}^{i}
\end{array}\right] \\
\cdot & & & \\
& \cdot & & \\
\sum \text { toeplitz } & \left(h^{i N r}\left[\begin{array}{llll}
x_{0}^{i} & x_{1}^{i} & \ldots . . ., & x_{N+N_{C P}}^{i}
\end{array}\right]\right.
\end{array}\right)
\end{aligned}
$$

Toeplitz function is a channel matrix function which can be defined as toeplitz $(\mathrm{h})=$

$$
\left(\begin{array}{cccccccc}
h^{i j}(0) & 0 & \cdots & 0 & 0 & 0 & 0 & 0 \\
h^{i j}(1) & h^{i j}(0) & 0 & \cdots & 0 & 0 & 0 & 0 \\
\vdots & h^{i j}(1) & h^{i j}(0) & 0 & \cdots & 0 & 0 & 0 \\
h^{i j}(l-1) & \vdots & h^{i j}(1) & \ddots & 0 & \cdots & 0 & 0 \\
0 & h^{i j}(l-1) & \vdots & \ddots & \ddots & 0 & \cdots & 0 \\
\vdots & 0 & h^{i j}(l-1) & \vdots & \ddots & \ddots & 0 & \vdots \\
0 & \vdots & \vdots & \ddots & \vdots & \ddots & \ddots & 0 \\
0 & 0 & 0 & 0 & h^{i j}(l-1) & \cdots & h^{i j}(1) & h^{i j}(0)
\end{array}\right)
$$

After removing cyclic prefix and FFT transformation by OFDM demodulator, received signal in frequency domain can be represented as

$$
Y_{k}^{j}=\sum_{i=1}^{M} H_{K}^{j i} \cdot S_{K}^{i}+W_{k}^{j}
$$

Wkj is AWGN in frequency domain and it can be calculated using

$$
W_{k}^{j}=\frac{1}{\sqrt{N}} \sum_{n=0}^{N-1} w^{j} \cdot e^{-j 2 \pi k n / N}
$$

Receive signal in vector form can be represented as

$$
\begin{aligned}
Y^{j} & =\sum_{i=1}^{M} S^{i} H_{K}^{j i}+W^{j} \\
& =\mathrm{S} . \mathrm{HJ}+\mathrm{WJ}
\end{aligned}
$$

In more detail each of variable in Equation (14) can be written as in Equations (15),(16),(17) and (18).

$$
Y^{j}=\left[Y_{1}^{j}(n), \quad y_{1,}^{j}, \ldots ., \quad y_{N}^{j}(n)\right]^{T}
$$




$$
\begin{gathered}
H_{K}^{j i}=\left(\left[H_{1}^{(1, J)}, H_{2}^{(1, J)}, \cdots, H_{N}^{(1, J)}\right], \cdots ;\left[H_{1}^{(M, J)}, H_{2}^{(M, J)}, \cdots, H_{N}^{(M, J)}\right)^{T}\right. \\
S=\left(\begin{array}{ccccccccc}
S_{1}^{1}(n) & 0 & \cdots & 0 & \cdots & S_{1}^{M}(n) & 0 & \cdots & 0 \\
0 & S_{2}^{1}(n) & \cdots & 0 & \cdots & S_{1}^{M}(n) & 0 & \vdots & 0 \\
\vdots & 0 & \ddots & \vdots & \vdots & \vdots & 0 & \ddots & \vdots \\
0 & \cdots & 0 & S_{N}^{1}(1) & \cdots & 0 & \cdots & 0 & S_{1}^{M}(n)
\end{array}\right) \\
W^{j}=\left[\begin{array}{lllll}
W_{1}{ }^{j}(n), & W_{2}{ }^{j}(n), & \cdots & W_{N}^{j}(n)
\end{array}\right]^{T}
\end{gathered}
$$

Received signal by antenna $[1,2 \ldots \mathrm{Nr}]$ in frequency domain after removing cyclic prefix and FFT transformation can be written as

$$
\mathrm{Y}=\mathrm{S} \times \mathrm{H}+\mathrm{W}
$$

In more detail each of variables in Equation (19) can be written as in Equations (17), (20), (21) and (22)

$$
\begin{aligned}
& Y=\left(\begin{array}{cccc}
Y_{1}^{1}(n) & y_{N}^{2}(n) & \cdots & y_{N}^{N_{r}}(n) \\
y_{2}^{1}(n) & y_{N}^{2}(n) & \cdots & y_{N}^{N_{r}}(n) \\
\vdots & \vdots & \vdots & \vdots \\
y_{N}^{1}(n) & y_{N}^{2}(n) & \cdots & y_{N}^{N_{r}}(n)
\end{array}\right) \\
& H=\left(\begin{array}{cccc}
H_{1}^{(1,1)} & H_{1}^{(1,2)} & \cdots & H_{1}^{\left(1, N_{r}\right)} \\
H_{2}^{(1,1)} & H_{2}^{(1,2)} & \cdots & H_{2}^{\left(1, N_{r}\right)} \\
\vdots & \vdots & \vdots & \vdots \\
H_{N}^{(1,1)} & H_{N}^{(1,2)} & \cdots & H_{N}^{\left(1, N_{r}\right)} \\
\vdots & \vdots & \vdots & \vdots \\
H_{1}^{(M, 1)} & H_{1}^{(M, 2)} & \cdots & H_{1}^{\left(M, N_{r}\right)} \\
H_{2}^{(M, 1)} & H_{2}^{(M, 2)} & \cdots & H_{2}^{\left(M, N_{r}\right)} \\
\vdots & \vdots & \vdots & \vdots \\
H_{N}^{(M, 1)} & H_{N}^{(M, 2)} & \cdots & H_{N}^{\left(M, N_{r}\right)}
\end{array}\right) \\
& W=\left(\begin{array}{cccc}
W_{1}^{1}(n) & W_{N}^{2}(n) & \cdots & W_{N}^{N_{r}}(n) \\
W_{2}^{1}(n) & W_{N}^{2}(n) & \cdots & W_{N}^{N_{r}}(n) \\
\vdots & \vdots & \vdots & \vdots \\
W_{N}^{1}(n) & W_{N}^{2}(n) & \cdots & W_{N}^{N_{r}}(n)
\end{array}\right)
\end{aligned}
$$

\section{LS CHANNEL ESTIMATION FOR MIMO-}

\section{OFDM}

From Equation (12), it can be seen that for estimation of channel component between receive antenna $\mathrm{j}$ and transmit antenna $\mathrm{i}=1,2, \ldots \mathrm{M}$, the number of subcarriers which has to be estimated is $\mathrm{M} \times \mathrm{N}$. where $\mathrm{N}$ is the number of subcarriers. In the other words for every receive antenna $\mathrm{j}=1,2, \ldots \mathrm{Nr}$ vector $H_{k}^{j i}$ in Equation(16) has to be estimated.
If one OFDM training block with $\mathrm{N}$ subcarriers transmitted from every of transmitted antenna, then from the model for every receive antenna there will be $\mathrm{N}$ equation with $\mathrm{N} \times \mathrm{M}$ unknown, hence these equations are under determined and cannot be solved. For solving this problem there are two solutions, first solution is transmitting M OFDM blocks which in practical case is not applicable. Second solution is reducing the unknown elements by looking at an alternate representation of the received signal, called the transformdomain estimator that was first proposed by van de Beek in [4] for OFDM systems and well explained in [2] for MIMOOFDM system. Base on this method CFR (Channel Frequency Response) can be expressed in terms of the CIR (Channel Impulse Response) through the Fourier transformation. Hence, the received signal model in "Equation (14)" can be expressed in terms of the CIR. The benefit of this representation is that usually the length of the CIR is much less than the number of subcarriers of the system. CIR representation can be achieved using following transformation

$$
H^{(j, i)}=F \cdot h^{(j, i)}
$$

Where $h(j, i)$ is the $(\mathrm{L} \times 1)$ channel impulse vector and $F$ is Fourier transform in vector form, and it can be represented as

$$
F=\left(\begin{array}{cccc}
1 & 1 & \cdots & 1 \\
1 & e^{\frac{-j 2 \pi(1)(1)}{N}} & \cdots & e^{\frac{-j 2 \pi(1)(L-1)}{N}} \\
\vdots & \vdots & \vdots & e^{\frac{-j 2 \pi(2)(L-1)}{N}} \\
1 & e^{\frac{-j 2 \pi(N-1)(2)}{N}} & \cdots & \vdots \\
1 & e^{\frac{-j 2 \pi(N-1)(1)}{N}} & \cdots & e^{\frac{-j 2 \pi(N-1)(L-1)}{N}}
\end{array}\right)_{N \times L}
$$

To extend the matrix Fourier transform to operate on multiple channels following matrix in Equation (25) can be defined as

$$
\Gamma=\left(\begin{array}{cccc}
F & 0 & \cdots & 0 \\
0 & F & \cdots & 0 \\
\vdots & \cdots & \ddots & \vdots \\
0 & \cdots & 0 & F
\end{array}\right)_{M \times D I M(F)}
$$

By using this definition, transformation of CFR to CIR in Equation (14) can be done as

$$
\begin{gathered}
Y^{j}=S^{j} H_{K}^{j}+W^{j}=X^{j} \cdot \Gamma \cdot h^{j}+W^{j} \\
A \cdot h^{J}+W^{j}
\end{gathered}
$$

By applying LS algorithm on Equation (26), channel component can be estimated using Equation (27). By this transformation one OFDM block is enough to estimate the channel. The only condition is $\mathrm{N} \geq \mathrm{M} \times \mathrm{L}$

$$
\tilde{h}^{j}=\left(A^{H} \cdot A\right)^{-1} \cdot A^{H} \cdot Y
$$




\section{QR DECOMPOSITION}

QR decomposition is just an alternative for calculating matrix inversion. There are different methods for QR decomposition. Here Householder algorithm is used. The steps of QRD algorithm to solve LS problem can be presented as follow [5]:

\section{Algorithm}

1. Making the LS error function for Equation (27) as represented in Equation (28).

$$
\varepsilon=Y-A \tilde{h} \text { And if } \varepsilon=0 \Rightarrow Y=A \tilde{h}
$$

2. Decompose $\mathrm{W}$ into Hermitian matrix $\mathrm{Q}$ and upper triangular matrix R using Householder algorithm as Equation (29).

$$
Y=A \tilde{h}=Q_{M \times M} \cdot\left[\begin{array}{l}
R \\
0
\end{array}\right]_{M \times N} \tilde{h}
$$

3. Multiply Hermitian of Q to both side of Equation (29). The result can be represented as in Equation (30).

$$
\left[\begin{array}{l}
R \\
0
\end{array}\right]_{M \times N} \cdot \tilde{h}=Q_{M \times M}{ }^{H} \cdot Y
$$

4. Finally, solve the channel using back substitution

\section{COMPLEXITY COMPARISON BETWEEN LS AND QRD ALGORITHM}

The advantage of using QR decomposition is to reduce the computational complexity of the LS channel estimation. In this research, the computational complexity in terms of number of mathematical operations has been measured. The derivations are based on an Mt-by-Mr MIMO-OFDM system with $\mathrm{N}$ subcarriers and a channel length of $\mathrm{L}$. The known matrix A has dimensions ( $\mathrm{N} \times \mathrm{L} . \mathrm{Mt})$. For simplicity in notation L.Mt is denoted by M. For a consistent comparison, the complex operations are converted to real operation equivalents.

Table 2 shows the real equivalent operations for the various complex operations. In addition, each type of real operations has different levels of complexity when implemented in the hardware. For example multiplications, additions, and subtractions can be set to 1 FLOPs (Floating Point Operations), divisions to 6 FLOPs, and square roots to 10 FLOPs (table-1). It should be emphasized that counting of the number operations is only an estimate of the computational complexity of the algorithms. A more exact measure would be to implement the algorithm in hardware and count the number of instructions and processing time required. However, in computer simulations, FLOP counts can give a good indication of the relative complexity of different algorithm.

Table-1: Number of flops in every real operation

\begin{tabular}{|l|l|}
\hline Operation & \# No of Flops \\
\hline $\begin{array}{l}\text { Multiplication, addition } \\
\text { and subtraction }\end{array}$ & 1 \\
\hline Division & 6 \\
\hline Square root & 10 \\
\hline
\end{tabular}

Table-2: Number of real operations in every complex operation

\begin{tabular}{|l|l|l|l|}
\hline \multirow{2}{*}{$\begin{array}{l}\text { Complex } \\
\text { Operation }\end{array}$} & \multicolumn{3}{|l|}{ No of Real Operations } \\
\cline { 2 - 4 } & $\begin{array}{l}\text { Multipli } \\
\text { cation }\end{array}$ & Division & $\begin{array}{l}\text { Subtraction } \\
\text { \& Addition }\end{array}$ \\
\hline Multiplication & 4 & 2 & 0 \\
\hline Division & 6 & 3 & 2 \\
\hline $\begin{array}{l}\text { Subtraction } \\
\text { /Addition }\end{array}$ & 0 & 0 & 2 \\
\hline $\begin{array}{l}\text { Complex } \\
\text { magnitude }\end{array}$ & 2 & 0 & 1 \\
\hline
\end{tabular}

\section{ITERATIVE QRD CHANNEL ESTIMATION}

In order to complete data-aided channel estimation, pilot signals can be spaced separated in the transmitted symbols. In the receiver, the channel impulse response can be estimated at the positions of pilot signals by several algorithms, such as least square method. The other channel information at the data signals can be obtained by interpolating the estimated channel impulse response [6].

In this paper, we propose a iterative LS-QRD channel estimation algorithm for MIMO OFDM system. At first step, an LS-QRD channel estimate is obtained by using (31)

$$
\hat{H}_{Q R D}^{j}=\Gamma \tilde{h}^{j}
$$

$\tilde{h}_{\text {can be obtained from }}\left[\begin{array}{c}R \\ 0\end{array}\right]_{M \times N} \cdot \tilde{h}=Q_{M \times M}{ }^{H} \cdot Y$

Where $\Gamma=\left[\begin{array}{cccc}F & F & \cdots & F \\ 0 & F & \cdots & 0 \\ \vdots & \vdots & \ddots & \vdots \\ 0 & 0 & \cdots & F\end{array}\right] \quad F=e^{-j 2 \pi n n / N}, \quad 0 \leq m \leq N-1,0 \leq n \leq L-1$

And then the channel estimate is set as initial $\hat{H}_{Q R D}^{j{ }^{(k)}}, k=0$.Secondly, the receiver uses the estimated channel to help the detection/decision of data signals. The detection data can be obtained by zero forcing method

$$
\hat{X}_{Z F}^{i}=\hat{H}_{Q R D}^{(k)}{ }^{\Phi} Y^{j}
$$


Where $\hat{H}_{Q R D}^{(k)}{ }^{\Phi}$ is pseudo inverse of $\hat{H}_{Q R D}^{j}$

$$
\hat{H}_{Q R D}^{(k)}=\left[\begin{array}{cccc}
\hat{H}_{Q R D, \text { diag }}^{1,1(k)} & \hat{H}_{Q R D, \text { diag }}^{2,1(k)} & \cdots & \hat{H}_{Q R D, \text { diag }}^{N_{t}, 1(k)} \\
\hat{H}_{Q R D, \text { diag }}^{1,2(k)} & \hat{H}_{Q R D, \text { diag }}^{2,2(k)} & \cdots & \hat{H}_{Q R D, \text { diag }}^{N r, 2(k)} \\
\vdots & \vdots & \vdots & \vdots \\
\hat{H}_{Q R D, \text { diag }}^{1, N_{r}(k)} & \hat{H}_{Q R D, \text { diag }}^{2, N_{r}(k)} & \cdots & \hat{H}_{Q R D, \text { diag }}^{N_{t}, N_{r}(k)}
\end{array}\right]
$$

And then the channel estimation treats the detected signals as known data to perform a next stage channel estimation iteratively and the index $\mathrm{k}$ adds 1 . Go to the first step and repeat the process till the mean-square-error of channel estimate is converged or the expected iterations reach. By utilizing the iterative channel estimation and signal detection process we can reduce the estimation error caused by channel interpolation between pilots. The accuracy of the channel estimation can be improved by increasing the number of iteration process.

\section{SIMULATION RESULTS}

The system specification for this simulation can be summarized in Table 3. For this simulation the channel has $\mathrm{L}=16$ paths where the amplitude of each path varies independently according to the Rayleigh distribution with an exponential power delay profile [10], and can be represented as in Equation (33). The results can be classified into two parts; Performance comparison and complexity comparison results. These are presented in the next sections.

\section{A. Iterative QRD Algorithm}

The bit error rate (BER) and MSE Performance of iterative QRD channel estimation method are shown in figure 2 and 3.Iteration number $\mathrm{r}=0$ means the conventional $\mathrm{QRD}$ channel estimation method. After about 2 iterations, the BER and MSE performance of iterative channel estimation are much closer to that of ideal one .

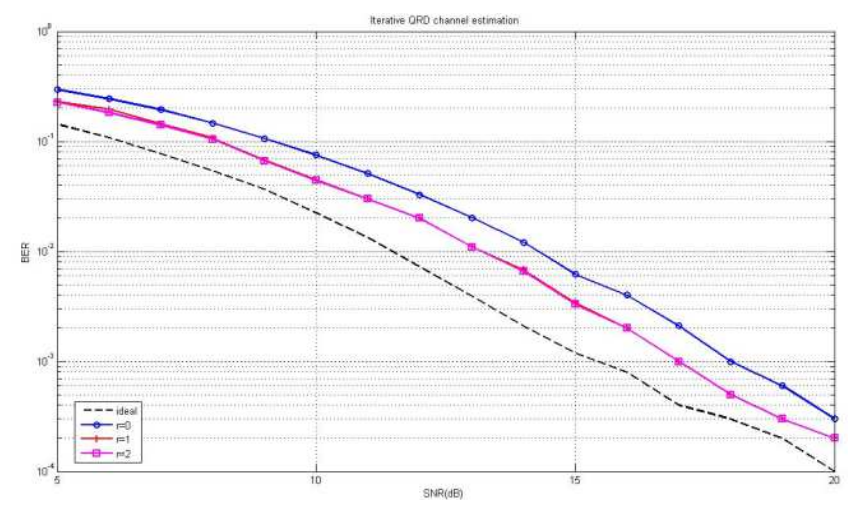

Figure-2: BER Performance of iterative channel estimation with different iteration numbers
Table-3: Simulation parameters for MIMO-OFDM

\begin{tabular}{|l|l|}
\hline System & MIMO(STBC)-OFDM \\
\hline \#Rx Antenna & 2 \\
\hline \#Tx Antenna & 2 \\
\hline Channel & $\begin{array}{l}\text { Frequency } \\
\text {,Rayleigh fading }\end{array}$ \\
\hline Noise & AWGN \\
\hline \#Sub carrier & 64 \\
\hline \#Cyclic Prefixes & 16 \\
\hline Cahnnel length & 16 \\
\hline Trms (RMS delay spread) & $25 \mathrm{~ns}$ \\
\hline Ts-Sampling Frequency & $1 / 80 \mathrm{MHz}$ \\
\hline
\end{tabular}

$$
h_{i}=N\left(0,1 / 2 \sigma^{2}\right)+j N\left(0,1 / 2 \sigma_{l}^{2}\right) \quad l=1,2, \ldots . L-1
$$

Where $\quad \sigma_{l}^{2}=\left(1-e^{\frac{-T_{s}}{T_{R M S}}}\right) \times e^{\frac{-L T_{s}}{T_{R M S}}}$ and ${ }_{\text {nor }}^{L-1} \sigma_{l}^{2}=1 \quad$ and $\mathrm{L}$ approximated by $\mathrm{L}=\frac{10 T_{r m s}}{T_{s}}$

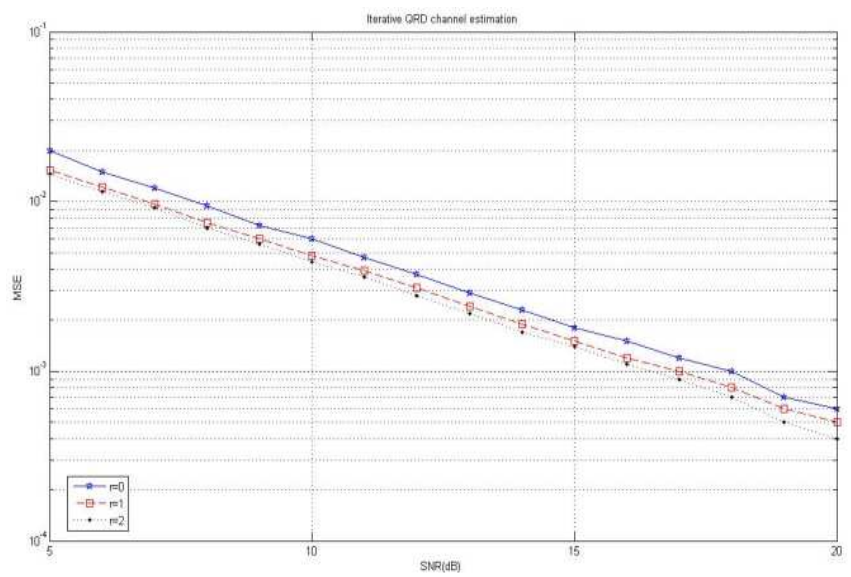

Figure-3: MSE Performance of iterative channel estimation with different iteration numbers.

The use of iterative QRD method improves the performance in terms of lower channel estimation error. From the results it can be concluded that the iterative QRD channel estimation algorithm have high performance efficiency in terms of BER and MSE. However, in the next section the benefit of QRD, which is the significant reduction of the complexity of the system, is portrayed.

\section{B. Complexity Comparison between LS and QRD Algorithm}

Using the system parameters for the MIMO-OFDM system specified in Table 3, the number of operations for a 2 transmit antenna system with a channel length of 6 and 16 was 
calculated for the two algorithms. In this section, the complexity comparison in terms of FLOPs count is performed for two algorithms. Figures 4 and 5 shows the complexity comparison of both algorithms

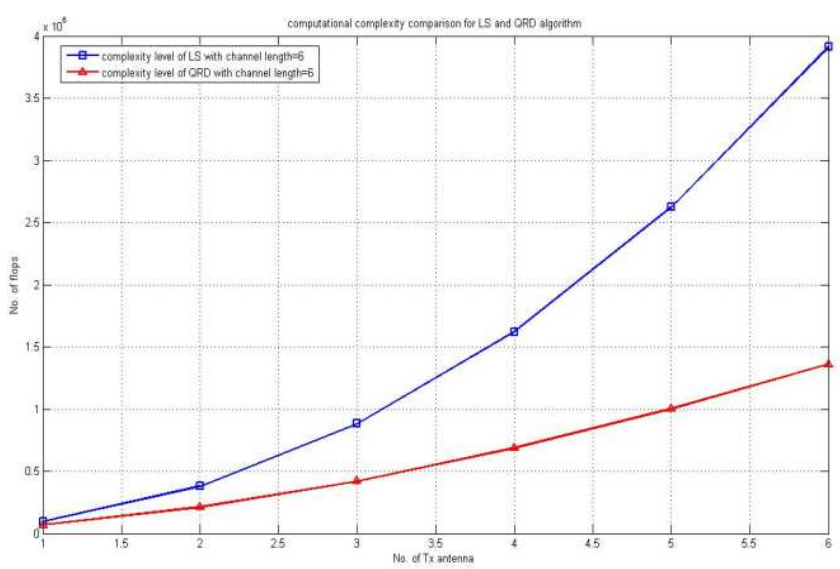

Figure-4: Complexity comparisons between LS and QRD with channel length $=6$

The results in Figure 5 is more highlighted which the number of channel length increase to 16 . Increasing the channel length increases the number of unknown parameters, thereby will increase the complexity of the channel estimation. It shows that the LS increases exponentially as the channel length increases and has much higher complexity than the QRD for long channel lengths

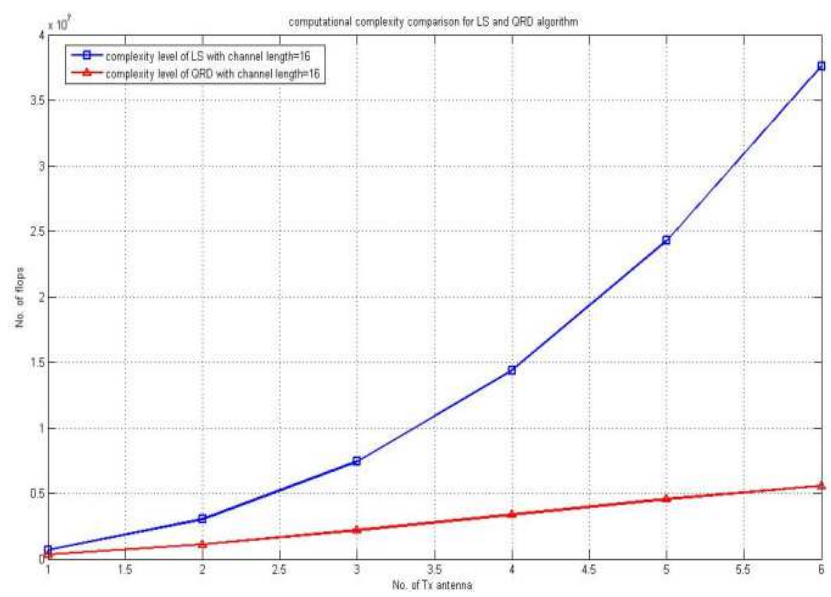

Figure-5: Complexity comparisons between LS and QRD with channel length $=16$

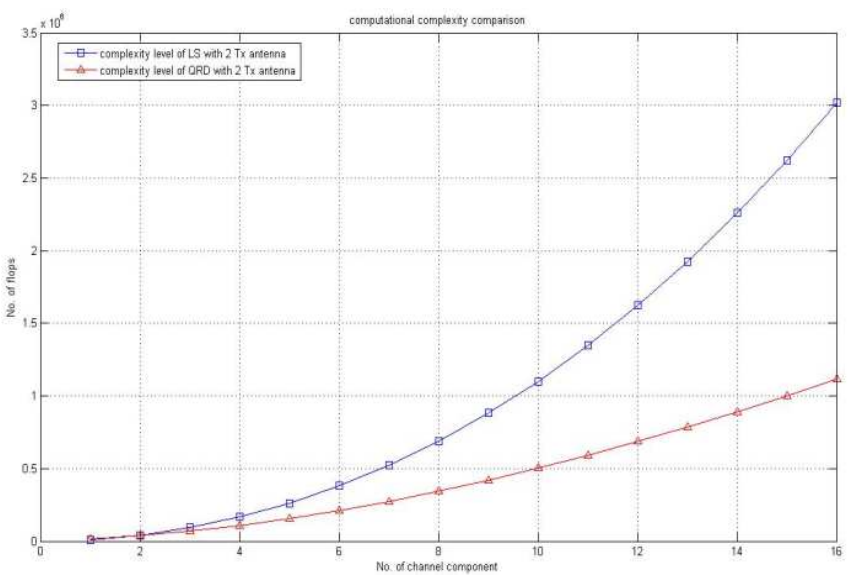

Figure-6: Complexity comparisons between LS and QRD with $\mathrm{TX}=2$

Figure 6 shows the simulation result using 2 transmit antenna while the number of channel component vary from 1 to 16 . The previous conclusion for computational complexity can be made here. In Figure 7, the number of transmit antenna is increased to 8 while the channel is changed from 1 to 16 . As expected, when the number of antennas increases, both estimation techniques increase in complexity because the size of the unknown matrix A increases. The general trend of the QRD method is that it increases almost linearly with the number of transmits antennas of the system. The LS method increases exponentially at a considerably higher rate than the QRD methods. Therefore, the QRD is especially preferable for higher number of transmit antennas since it does not explode in complexity as the LS solution. Finally the Numerical example for computational complexity comparison between two channel estimation algorithms is provided in Table 4.

Table-4: Number of complex operations and Flops in two algorithms with $\mathrm{L}=16$ and $\mathrm{Tx}=4$ (A: \# of complex ultiply, B: \# of add/sub, C: \# of complex division, D: \# of square root, E: \# of complex magnitude, F: \# of flops).

\begin{tabular}{|l|l|l|l|l|l|l|}
\hline $\begin{array}{l}\text { Algorith } \\
\mathrm{m}\end{array}$ & A & B & C & D & E & F \\
\hline LS & $\begin{array}{l}79052 \\
8\end{array}$ & $\begin{array}{l}782277 \\
2\end{array}$ & $\begin{array}{l}617 \\
6\end{array}$ & 0 & 0 & $\begin{array}{l}1437356 \\
8\end{array}$ \\
\hline QRD & $\begin{array}{l}18722 \\
0\end{array}$ & $\begin{array}{l}184047 \\
1\end{array}$ & 192 & $\begin{array}{l}214 \\
4\end{array}$ & $\begin{array}{l}12 \\
8\end{array}$ & 3376126 \\
& 0 & & 4 \\
\hline
\end{tabular}

The results prove that QRD method is lower in complexity than LS method. The results in Table 4 show that the total number of operation for the LS method is much higher than the QRD method. For this simulation scenario using QRD achieves a complexity reduction by approximately $77 \%$. This verifies that the QRD has significantly lower complexity than that of direct LS estimation via the pseudo inverse, hence a better option for channel estimation. 


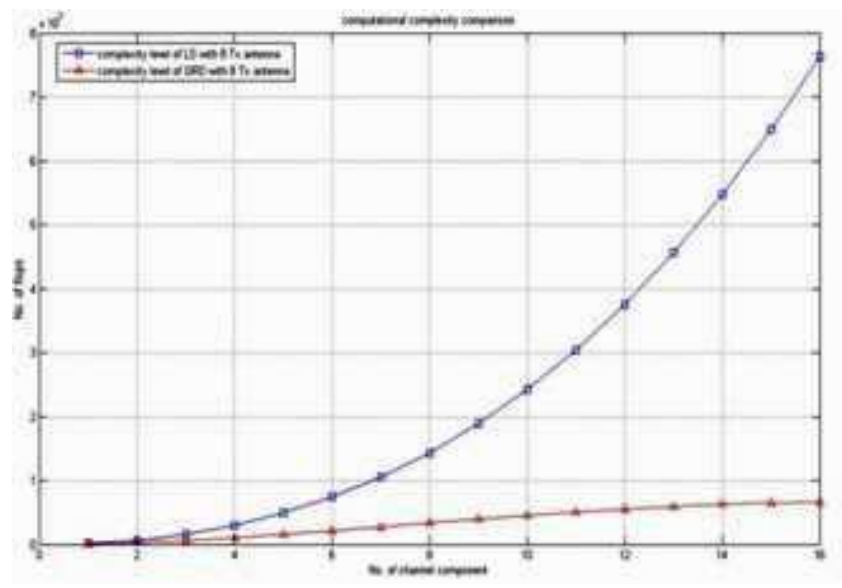

Figure-7: Complexity comparisons between LS and QRD with $\mathrm{TX}=8$

\section{CONCLUSIONS}

The simulation results proved that Iterative QRD channel estimation algorithm has good performance efficiency it can provide better mean square error and bit error rate performance than conventional methods. However the computational complexity of the QRD channel estimation is much lower than LS algorithm. In addition, computational complexity for QRD channel estimation is approximately linearly proportional with number of transmit antenna and channel length, whereas for LS algorithm is exponentially proportional with the number of transmit antenna and channel length. As finding indicate; using QRD channel estimation, computational complexity of the system for above particular scenario which mentioned in table- 4 can dramatically decrease by $77 \%$.

Finally it can be concluded that Iterative QR decomposition can be an ultimate solution for high performance efficiency and reduction computational complexity.

\section{REFERENCES}

[1].G. L. Stuber, J. R. Barry, S. W. Mclaughlin, Y. Li, M. A. Ingram, and T. G. Pratt, "Broadband MIMO-OFDM wireless communications," Proceedings of IEEE, vol. 92, no. 2, pp. 271-294, Feb. 2004.

[2] T. Y. Al-Naffouri, "Adaptive algorithms for wireless channel estimation," Department of Electrical Engineering, Stanford University, Dec. 2004, PHD thesis.

[3] Kathryn Kar Ying Lo "Channel Estimation of Frequency Selective Channels for MIMO-OFDM" University og Calgary. Master thesis 2005

[4]. J.-J. van de Beek, O. Edfors, M. Sandell, S.K. Wilson, and P.O. Borjesson, "On channel estimation in OFDM system," in Proceedings of IEEE Vehicular Technology Conference 1995, vol. 2, pp. 815-819, Sept. 1995.
[5]Computational Complexity Reduction for MIMO-OFDM Channel Estimation Algorithms Reza Abdolee Tharek Abd.Rahman Sevia Mahdaliza Idrus

VOL. 9, NO. 1, 2007, 30-36 elektrika Universiti Teknologi Malaysia

[6]. Ju-Ya Chen, Cheng-Chih Chiang, and Li-Chung Lo"Iterative Channel Estimation for MIMO OFDM Systems", Proceedings of 2007 International Symposium on Intelligent Signal Processing and Communication Systems Nov.28Dec.1, 2007 Xiamen, China

[7].Tarokh, V., Jafarkhani, H., and Calderbank, A. R., "Spacetime block codes from orthogonal designs," IEEE Trans, Inform. Theory, Vol. 45, No. 5, pp. 1456-1467, July 1999.

[8]. Y. Qiao, S. Yu, P. Su, and L. Zhang, "Research on an iterative algorithm of LS channel estimation in MIMO OFDM systems," IEEE Trans. Broadcast., vol. 51, pp. 149-153, Mar.2005.

[9]. I. Barhumi, G. Leus, and M. Moonen, "Optimal training design for MIMO OFDM systems in mobile wireless channels," IEEE Trans. Signal Proc., pp. 1615-1624, June 2003.

[10].Part 11: Wireless LAN Medium Access Control (MAC) and Physical Layer (PHY) Specifications: High-speed Physical Layer in $5 \mathrm{GHz}$ Band, IEEE Std 802.11a1999(R2003). 